\title{
Self-excited Contact Resonance Operation of a Tactile Pie- zoresistive Cantilever Microprobe with Diamond Tip
}

\author{
Michael Fahrbach ${ }^{1}$, Min X $\mathrm{U}^{2}$, Uwe Brand ${ }^{2}$ and Erwin Peiner ${ }^{1}$ \\ 1 Technische Universität Braunschweig, Institute of Semiconductor Technology (IHT), Hans-Sommer- \\ Straße 66, 38106 Braunschweig, Germany and Technische Universität Braunschweig, Laboratory for \\ Emerging Nanometrology (LENA), Langer Kamp 6A/B, 38106 Braunschweig, Germany \\ 2 Physikalisch-Technische Bundesanstalt (PTB), Bundesallee 100, 38116 Braunschweig, Germany \\ m.fahrbach@tu-bs.de
}

\section{Summary:}

In this work, a self-exciting tactile cantilever sensor for contact resonance applications is described. The design integrates heating resistors with the aim to enable high-speed measurements on large workpieces. For this purpose, higher-order out-of-plane bending modes of the cantilever are favorable. Consequently, the position of the actuators is optimized using finite-element modelling to achieve uniform signal amplitudes for a range of materials when using the third vibration mode. Preliminary measurements with thin polymer films show the basic function of the design.

Keywords: MEMS, microprobe, thermal actuator, piezoresistive cantilever, contact resonance

\section{Motivation}

In industrial environments, where surfaces can be covered with contaminants like lubricants or cooling liquids, monitoring of workpieces using optical techniques is often difficult or not possible. Here, piezoresistive microprobes with integrated silicon tips are promising as they can scan surfaces at velocities up to $15 \mathrm{~mm} / \mathrm{s}$ [1]. However, their silicon tip quickly wears down during measurements and, if mechanical characterization of the sample using contact resonance (CR) techniques are to be performed, external actuators are necessary.

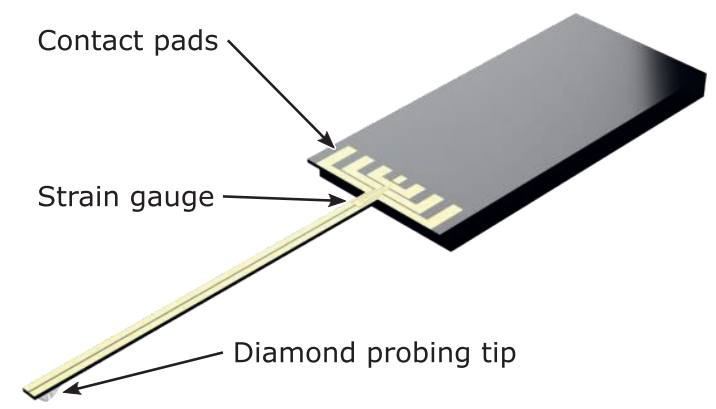

Fig. 1. Render of the microprobe design.

The European EMPIR project MicroProbes is developing new sensors, that aim to improve on the shortcomings of previous designs [2]. These new sensors shall be able to efficiently measure mechanical properties using CR. Then, they shall be calibrated on reference samples.

\section{Design of the microprobe}

The basic shape of our new probes is based on previous designs [3], with the cantilevers being 5 $\mathrm{mm}$ long, $200 \mu \mathrm{m}$ wide and $50 \mu \mathrm{m}$ thick. As shown in Fig. 1, the probes integrate a strain gauge near the clamped end of the beam and contact pads close to the edge of the base. Additionally, wear resistant diamond probing tips are included close to the free end of the beam and, as shown in Fig. 2, heating resistors are distributed across the length of the cantilever

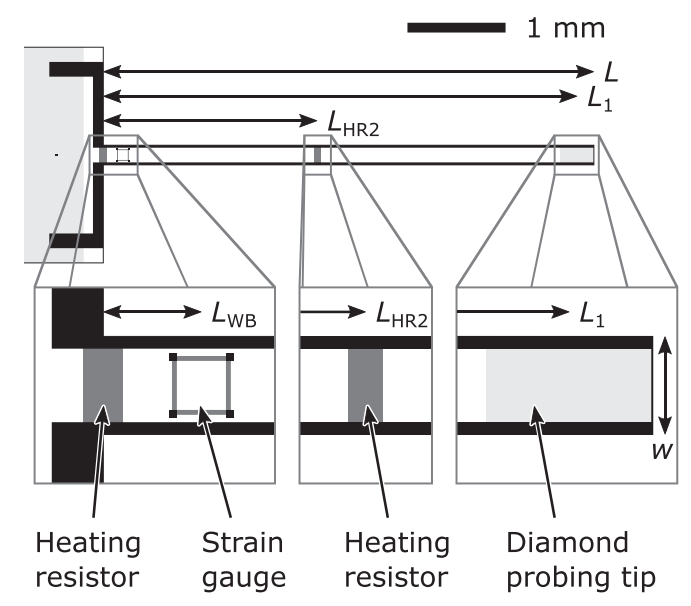

Fig. 2. Overview of the microprobe design. The metal contact lines are not shown to maintain visibility.

Previous measurements using a commercial CAN50-2-5 probe (CiS Forschungsinstitut für Mikrosensorik GmbH, Erfurt, Germany) [4] are used to evaluate the performance of different $\mathrm{CR}$ vibration modes. Here, the third flexural vibration 
mode at $53 \mathrm{kHz}$ is a good compromise of resolution, measurement speed and ease of use.

To actuate this vibration mode more efficiently, this design is changed by two heating resistors placed on the cantilever. Their locations are optimized to enable uniform signal amplitudes when analyzing a large range of materials [5].

The strain gauge is located close to the clamping at $L_{\mathrm{WB}}=185 \mu \mathrm{m}$ and distanced from the adjacent heater by $100 \mu \mathrm{m}$ to minimize parasitic thermal coupling [6].

\section{FEM simulations of contact mechanics}

After completing the sensor design, finite element modelling (FEM) simulations of the sensor in contact with the surface of a sample are performed using COMSOL 5.4. As shown in Fig. 3, large frequency shifts are expected for the first three CR-modes when analyzing samples with Young's moduli up to $10 \mathrm{GPa}$. Consequently, compliant layers on stiff substrates, such as polymers on silicon, can be measured with high resolution.

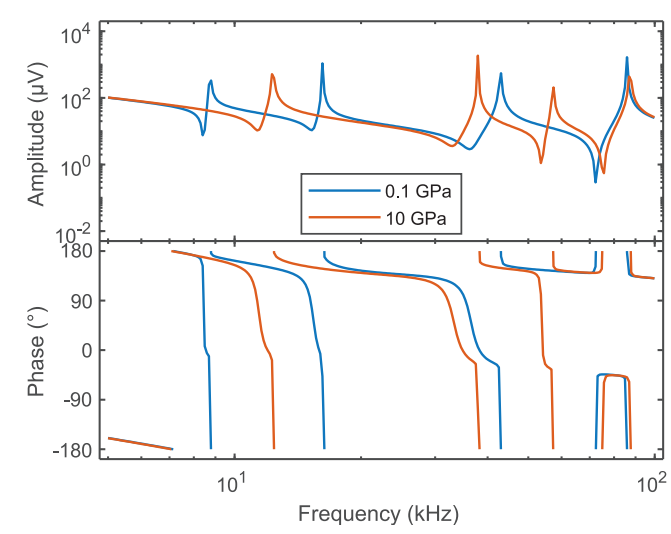

Fig. 3. FEM frequency response of the strain gauge output for sample Young's moduli of $E=0.1 \mathrm{GPa}$ and $E=10 \mathrm{GPa}$.

\section{Fabrication and Test}

First sensor samples with a thickness of approximately $100 \mu \mathrm{m}$ are fabricated. Due to the increased thickness, the measured resonance frequencies of these sensors are approximately 2.5 times higher than calculated using FEM. One of these probes is shown in as shown in Fig. 4.

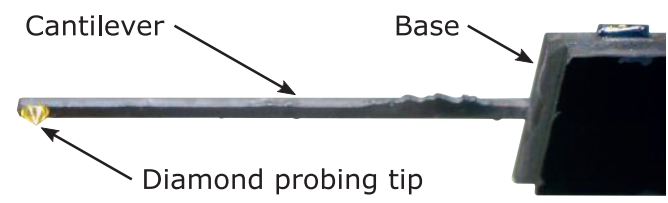

Fig. 4. Photograph of the bottom side of a prototype microprobe.

Then, preliminary measurements on a thin polymer film are conducted. The results are shown in Fig. 5. Here, CR spectra are acquired at different probing forces on the same sample. As expected, the CR frequency increases with increasing probing force.

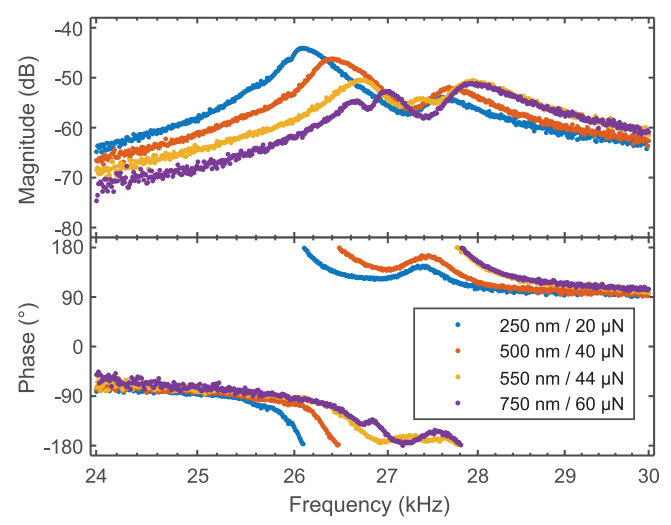

Fig. 5. Measured fundamental mode CR frequency responses on a thin polymer film for different probing forces given by the respective cantilever deflections.

As the next step, measurements on reference samples will be performed to calibrate the microprobes for enabling a quantitative analysis.

\section{Acknowledgements}

This project has received funding from the EMPIR programme co-financed by the participating states and from the European Union's Horizon 2020 research and innovation programme under no. 17IND05 MicroProbes.

\section{References}

[1] H. S. Wasisto et al., Ultra-high-speed cantilever tactile probe for high-aspect-ratio micro metrology, 2015 Transducers - 2015 18th International Conference on Solid-State Sensors, Actuators and Microsystems (TRANSDUCERS). IEEE. (2015); doi: 10.1109/transducers.2015.7181109

[2] 17IND05 MicroProbes Multifuntional ultrafast microprobes for on-the-machine measurements. (2018). Retrieved from https://www.ptb.de/empir2018/microprobes/

[3] E. Peiner et al., Slender Tactile Sensor for Contour and Roughness Measurements Within Deep and Narrow Holes, IEEE Sensors Journal 8, 1960-1967 (2008); doi: 10.1109/jsen.2008.2006701

[4] M. Fahrbach et al., Calibrating a high-speed contact-resonance profilometer Journal of Sensors and Sensor Systems vol. 9, no. 2, Art. no. 2 (2020); doi: 10.5194/jsss-9-179-2020.

[5] M. Fahrbach, E. Peiner, Higher-Mode Contact Resonance Operation of a High-Aspect- Ratio Piezoresistive Cantilever Microprobe, SMSI 2020 Sensors and Instrumentation (2020); doi: 10.5162/SMSI2020/A6.3.

[6] A. Setiono et al., Phase optimization of thermally actuated piezoresistive resonant MEMS cantilever sensors. Journal of Sensors and Sensor Systems 8, 37-48 (2019); doi: 10.5194/jsss-8-37-2019 